\title{
Factors contributing to adolescents' COVID-19-related loneliness, distress, and worries
}

\author{
Christa C. Christ ${ }^{1}$. Jennifer M. Gray ${ }^{1}$
}

Accepted: 19 January 2022

(c) The Author(s), under exclusive licence to Springer Science+Business Media, LLC, part of Springer Nature 2022

\begin{abstract}
As the world continues to face uncertainty due to COVID-19, there is concern for adolescent wellbeing as they attempt to cope with changes to their education and social interactions. Considering the trade-offs between physical distancing and virtual education versus in-person instruction may be particularly pertinent to understanding adolescent loneliness and worries surrounding COVID-19 and their academics. Adolescents $(N=1,708$; age $M=12.42, S D=2.40 ; 53.3 \%$ girls and $46.7 \%$ boys) from the United States self-reported their perceptions of social support, COVID-19 attitudes, and indicators of wellbeing (i.e., loneliness, COVID-19 stress, academic worry) in an online cross-sectional survey between November 30, 2020 to December 21, 2020. Higher levels of social support and attitudes of lower COVID-19 concern were associated with lower levels of loneliness, COVID-19 stress, and academic worries. Adolescents receiving greater virtual instruction were more worried about their academics compared to those receiving greater in-person instruction. A moderation effect between loneliness and instruction type revealed that the effect of social support was stronger for those receiving greater virtual instruction. Despite the quick and dramatic change to one's education, adolescents were relatively unaffected by the type of instruction they had recently received. Instead, perceived social support and their attitudes toward COVID-19 were more strongly related to their wellbeing.
\end{abstract}

Keywords Teaching method $\cdot$ Adolescence $\cdot$ Stress $\cdot$ Academics $\cdot$ Loneliness $\cdot$ Pandemic

\section{COVID-19 Reactions and Mental Health}

COVID-19 was first reported in December 2019 and became a pandemic by March 2020. By February 2021, the pandemic had resulted in over 110 million cases and 2.4 million deaths worldwide (World Health Organization, 2021). The pandemic can be characterized as a period of high unpredictability and uncertainty that many are experiencing for the first time. With this unique experience, individuals are reporting higher rates of mental health issues, such as anxiety and depression (Rogers et al., 2021; Wu et al., 2021). Particularly, younger individuals report greater difficulties with their mental health (Lahav, 2020; Shah et al., 2021; Taylor et al., 2020) despite the higher mortality rates for older individuals (Center for Disease Control, 2021a). Feelings of fear regarding the spread of COVID-19 have been

Christa C. Christ

cchrist@uscupstate.edu

1 Department of Psychology, University of South Carolina Upstate, 800 University Way, Spartanburg, SC 29303, USA shown to be positively correlated with loneliness among adolescents (Ellis et al., 2020). Among adults, greater worry about COVID-19 was associated with increased loneliness (Kotwal et al., 2021); although other studies have found no association (Grossman et al., 2021). Feelings of fear of COVID-19 is also associated with higher perceived risk of COVID-19 (Breakwell \& Jaspal, 2020; Harper et al., 2020).

As dangerous as COVID-19 is, fear of the virus may only partly explain mental health changes during the pandemic. One additional contributor to the increase in anxiety and depression is the loneliness resulting from physical distancing efforts meant to reduce the spread of COVID-19 (Lee et al., 2020; Ruggieri et al., 2021). Adolescents assessed between March and August 2020 indicated that physical distancing increased distress and lowered happiness (Munasinghe et al., 2020). Research beyond this pandemic has also indicated that the effects of isolation, and particularly the duration of loneliness, on mental health can be long-lasting (Loades et al., 2020). Therefore, in order to determine if physical distancing during the COVID-19 pandemic is also 
long-lasting, research examining mental health at regular intervals throughout the pandemic is necessary.

\section{Protective Factors}

The buffering model of social support holds that perceived social support is a protective factor when experiencing stress (Cohen \& Willis, 1985). Since they proposed this theoretical model, numerous empirical articles have found support in different contexts where stressors were present (e.g., children's ability to cope when their lives were impacted by the AIDS epidemic in Southern Africa; Barenbraum \& Smith, 2016). In the context of COVID-19, social support has been shown to be a protective factor against loneliness (Bu et al., 2020; Groarke et al., 2020; Li et al., 2021; Magson et al., 2021). One study demonstrated that a stronger social network had an inverse effect compared to perceived risk of COVID-19, where the stronger the social network, the lower the reported feelings of loneliness, anxiety, and depression; however, these feelings were higher the greater the perceived risk of COVID-19 (Jaspal \& Breakwell, 2020). Additionally, resiliency in adolescence, promoted through high social support from parents, peers, and school (Malhi et al., 2019; Weine et al., 2014), may facilitate coping with pandemic stressors more effectively. For example, one study found that children and adolescents experiencing stress due to the pandemic were more likely to seek social support from their peers and families. Additionally, they reported engaging in more positive stress reduction behaviors when they returned to face-to-face instruction (Zhu et al, 2021). Adolescents who engaged in problem-based coping strategies, such as seeking social support, were more likely to be well-adjusted after 1-month of online learning due to the pandemic ( $\mathrm{Li}$ et al., 2021).

Although this evidence suggests social support is a protective factor, the ability to access social support may be limited when adolescents are having to physically distance themselves from peers and family members outside the home. Based on the individual approach taken by schools to return to in-person instruction in the United States, some adolescents received in-person instruction, some received hybrid instruction, and some continued to learn virtually for the school year. Therefore, those who experienced greater physical distancing during the pandemic, and did not have sufficient social support, may have found it particularly challenging to remain resilient due to reduced access to these forms of social support. However, such a moderating effect between instruction type (e.g., virtual vs. in-person) and social support has not yet been tested. Social support can come from a variety of areas in one's life including family, friends, and teachers/mentors. However, for adolescents, peers are especially important to buffer against loneliness and stress during times of transition (e.g., elementary to middle school or middle to high school; Kingery et al., 2011; Virtanen et al., 2020). After experiencing a stressor, having contact with a peer decreased reported sadness more than contact with family (Uink et al., 2017). Although peers play a growing role in the emotional life of adolescence, familial support does remain important for adolescent mood (Cavanaugh \& Buehler, 2015). Peer and parental support offer unique contributions to lowering an adolescent's perceived loneliness (Cavanaugh \& Buehler, 2015). Further research demonstrates that when peer support is limited, parental support can provide a compensatory role in wellbeing (Schacter \& Margolin, 2019). Therefore, while parental and peer support may be effective at mitigating the impact of the pandemic, it may be that different types of support are effective to differing extents.

The stage of adolescent development may further provide context for understanding the impact of social support during the pandemic. In general, autonomy, self-efficacy, and independence increase during adolescence. As adolescents' independence increases, they spend more time with peers and less time with family. Adolescents also disclose more intimate information with peers as they develop emotional autonomy from their family as a form of social reorientation (Nelson et al., 2016), becoming less likely to confide in their parents as they transition from childhood to adulthood (Vijayakumar \& Pfeifer, 2020). Older adolescents report more parent-adolescent conflict (Steinberg, 2005). This conflict may be important for redefining familiar roles thus facilitating social reorientation (Nelson et al., 2016). The increased reliance on peers for social support as adolescents move into later stages of adolescence (Kingery et al., 2011; Vijayakumar \& Pfeifer, 2020) may contribute to increased stress and loneliness resulting from the COVID-19 pandemic for those remaining in a virtual learning environment as opposed to younger children remaining in the virtual learning environment. Because adolescence is a time period during which individuals begin to rely more heavily on their peers than family, physical distancing may be particularly adverse for this age group. However, there is limited research exploring the role of peers and family on adolescent wellbeing during the COVID-19 pandemic.

In addition to social support buffering distress surrounding the pandemic, it may be that certain social privileges buffer distress as well. The pandemic has highlighted social inequities for race (Chowkwanyun \& Reed, 2020), socioeconomic status (Clouston et al., 2021), and gender (Gausman \& Langer, 2020) for adults. It stands to reason than that, adolescents who are minorities, women, or living in poverty may be at greater risk for increased stress resulting from the pandemic than adolescents with social privileges (e.g., white, male, and affluent). Yet, little is currently known about the combined buffering effect of social support and 
social privileges on adolescent mental health and wellbeing during the COVID-19 pandemic.

\section{Educational Experience During the COVID-19 Pandemic}

With states initiating stay-at-home orders in mid-March 2020, the spring semester shifted to virtual learning. Adolescents in isolation during the COVID-19 pandemic indicated concern for their academics, with over $50 \%$ reporting their learning was affected (Duan et al., 2020). In fact, challenges related to their academics, mental health, and friends, were rated significantly greater than COVID-19 concerns (Scott et al., 2021). Although meta-analyses have indicated that virtual learning is equivalent to traditional instruction (Cavanaugh, 2001; Shachar \& Neumann, 2003), a key difference of the pandemic is that educators and students, who were inexperienced with virtual learning, were given no choice and little time to transition to this educational method, potentially hindering student learning while they simultaneously battle economic and personal hardships (American Psychological Association, 2021).

Although, COVID-19 cases continued to rise throughout the summer of 2020, schools announced they would attempt to transitions back to in-person teaching starting in the fall 2020 semester with hybrid approaches (alternating days of virtual and in-person) being used to provide physical distancing (AP News, 2021). Potentially suggesting that the concern about exposing adolescents to COVID-19 by transitioning back to in-person teaching was not as great as concerns about virtual education decreasing the ability to learn and the continued isolation from peers increasing distress. Therefore, with schools moving back into some version of in-person teaching in the fall of 2020, we might expect to see feelings of loneliness and academic stress decrease among adolescents at that time. Limited research has examined the role of education instruction method, social support, and mental health outcomes in children and adolescence. Of the research that has been conducted, most has either focused on children and adolescents who have returned to in-person instruction (Zhu et al., 2021) or who are engaged in online learning (Li et al., 2021; Magson et al., 2021). Adolescents who reported high levels of satisfaction with online learning were more likely to be well adjusted and experience less distress than their peers who reported lower levels of satisfaction with online learning (Li et al., 2021). Similarly, adolescents who experienced difficulties with online learning were more likely to report decreased mental health (Magson et al., 2021). Each study has expanded our understanding of how school provides access to protective factors or increases risk factors for the impact of COVID-19 on mental health. However, questions remain about protective factors and adolescents' distress during virtual learning in the United States.

\section{Study Purpose and Hypotheses}

While previous studies have demonstrated the importance of educational instruction and social support on mental health outcomes for adolescents during the COVID-19 outbreak, no study has occurred in the United States with children and adolescents to directly examine the ability of protective factors to moderate one's distress and loneliness resulting from online vs. in-person educational instruction and COVID-19 attitudes. The purpose of the current study is primarily to examine the contribution of education instruction type, attitudes toward COVID-19, and protective factors on adolescent mental health (i.e., loneliness, academic performance worry, and distress related to COVID-19) in the United States.

Specifically, we hypothesize that a latent trait of protective factors will be defined by both higher social support (from family, friends, and school) and social privileges (i.e., white, male, affluent). Because of the developmental difference across late childhood/early adolescence, middle adolescence, and late adolescence, we expect that higher family support will be perceived by early adolescence whereas higher friend support will be perceived by late adolescence. Therefore, the relationship between age and social support will be examined to investigate if hypotheses should be tested in each stage of adolescence separately. Then, when testing a full model with all variables, we hypothesize that adolescents receiving in-person instruction will be less lonely, less stressed about COVID, and less worried about their academics compared to adolescents receiving online/virtual instruction and that this effect will be especially prominent if adolescents have high levels of protective factors or are generally unphased by COVID-19 (e.g., holding attitudes that COVID-19 isn't harmful, not important to follow rules).

A secondary goal of this research is to provide insight into adolescent mental and emotional health during the point in the COVID-19 pandemic in which a full semester of schooling has been completed and roughly a year has passed since the first reported COVID-19 cases. Therefore, descriptive information about student attitudes, exposure, and feelings are presented. Continued collection of such descriptive data at regular intervals in the pandemic is critical in evaluating the progression of experiences and the duration of the effects of the pandemic. 


\section{Method}

\section{Participants}

Participants were adolescents from local public schools across several school districts in the Southeast United States. Assistant superintendents and directors of student services were contacted via email and provided information regarding the purpose of the study to solicit participation. Districts that participated were provided with the link for the electronic survey, which was distributed to individual schools at their discretion during the last 3 weeks of the semester prior to their winter break. Districts were also provided an opt-out letter to give to parents of adolescents 1-2 weeks prior to data collection, with the expectation that if teachers received a signed opt-out letter from a parent of an adolescent, they should not provide that adolescent with the study survey link. No report was made by teachers regarding how many parents chose to opt-out their child(ren). The survey began with an assent process in which participants had the option to take or opt-out of the electronic survey. Our institutional review board (IRB) approved the study.

The sample consisted of adolescents $(N=1,708)$, ages 9-19 $(M=12.42, S D=2.40 ; 53.1 \%$ late childhood/ early adolescence, $33.3 \%$ middle adolescence, $13.6 \%$ late adolescence). Additional demographic information is included in Table 1. The original sample contained 2,230 participants, but 522 were excluded due to the following: the participant did not (1) pass at least two of the three attention check questions (e.g., "You're doing great! Take a deep breath and select the option for yellow."; $N=117$ ), (2) complete at least $75 \%$ of the survey $(N=346)$, or (3) provide a response to critical questions (i.e., instruction method) or plausible responses to certain questions (i.e., age response higher than 19, suggesting a teacher/parent may have completed the survey; $N=59$ ).

\section{Measures}

Due to needing a simple and brief survey given the age range of our participants and the fact that the majority of validated measures related to COVID-19 were unavailable at the time of our data collection, most variables in our study were measured with single items. However, these single items were similar to items on validated measures made available after our data collection occurred and other studies involving adolescent samples (Dumas et al., 2020; Graupensperger et al., 2020; Murata et al., 2020; Rogers et al., 2021; Taylor et al., 2020).
Table 1 Demographic characteristics of included participants

\begin{tabular}{lll}
\hline Characteristic & $\mathrm{N}$ & $\%^{\mathrm{a}} / \mathrm{M}(\mathrm{SD})$ \\
\hline Gender & & \\
$\quad$ Boy & 792 & $46.4 \%$ \\
$\quad$ Girl & 910 & $53.3 \%$ \\
Race/Ethnicity & & \\
$\quad$ Black/African American & 296 & $17.3 \%$ \\
$\quad$ White/Caucasian & 1,276 & $74.7 \%$ \\
$\quad$ Hispanic/Latinx & 130 & $7.6 \%$ \\
$\quad$ Asian & 36 & $2.1 \%$ \\
$\quad$ Native American/American Indian & \\
$\quad$ Middle Eastern/North African & 129 & $7.6 \%$ \\
$\quad$ Native Hawaiian/Pacific Islander & 16 & $0.9 \%$ \\
Household Size & 12 & $0.7 \%$ \\
Percentage of Low-Income Students ${ }^{\mathrm{d}}$ & 1,636 & $0.70(0.44)$ \\
Instruction Method & 1,682 & $67.4 \%(12.3 \%)$ \\
$\quad$ All Virtual & & \\
$\quad$ Virtual/In-Person Hybrid & 64 & $3.7 \%$ \\
$\quad$ All In-Person & 718 & $42.1 \%$ \\
\hline
\end{tabular}

a Percentages for categorical variables will not add up to $100 \%$ because some participants chose not to report information and race/ ethnicity allowed for the selection of multiple options.

${ }^{b}$ Proportion of this racial group was higher than expected based on public reports from the school. This may be due to participants not understanding the category and/or the fact that they could select multiple racial groups rather than the one the most strongly identify with.

${ }^{c}$ Household size was used as a measure of SES and was calculated as the number of people living in the household / the number of rooms (bedrooms, bathrooms, kitchens, living rooms) in the household. Participants who reported living in two households provided information for both and the average household size of the two households reported was taken for those individuals.

d Percentage of low-income students was calculated by factoring the number of students who participated in the study from each school with the overall percentage of pupils in poverty as reported by the school. It should be noted that the accuracy of this measure is dependent on our sample being representative of the entire school's population.

\section{COVID-19 Exposure and Attitudes}

Instruction type being received by adolescents was determined with the item "How have you been attending school the last couple of months?" (1 - All virtual/Online, 2 - Hybrid/Some online and some in-person, 3- All in-person). Single items measuring exposure to COVID-19, behavior regarding COVID-19 precautions, and impressions of how harmful COVID-19 is were informed by COVID-19 surveys administered by Generation Scotland (2020). These items were "How much has COVID-19 changed how you usually spend your day?" (1 - A lot, to 4, Not at all), "How many times do you leave your home?" (1 - Many times per day, to 5, Less than once per week), "When leaving your home, do you do something with someone who does not live with 
you?" (1 - I don't leave my home, to 6, I seem someone every time I go out), "COVID-19 is harmful to everyone." (1 - A great deal, to 5, Not at all), "I have to follow rules (wear a mask, stay 6ft apart, etc.) to keep people from getting COVID-19." (1 - A great deal, to 5, Not at all), and "It has become harder to stay healthy because of COVID-19." (1 - A great deal, to 5, Not at all). These items were used to create a latent trait of adolescents' perceptions regarding behaviors to remain protected from COVID-19 exposure. A few more items were included for descriptive purposes: (1) "Have you or someone you lived with had COVID-19?" with response options "Yes, the doctor tested it", "Yes, we think so because of how sick someone felt", and "No" and (2) "How likely do you think it is that someone you live with will get COVID?" with response options "Someone will for sure", "Someone probably will”, "Someone could, but they probably won't", and "Not at all likely".

\section{Family, Friend, and School Support}

The Perceived Social Support/Conflict Scale was used in the Midlife Development in the U.S. (MIDUS) study (National Institute of Aging, 2002) and is used to measure both positive and negative interactions with family members (10-items) and friends (8-items). Positive interaction items (e.g., "How much do they understand the way you feel about things") were measured on a 4-point scale (from 1, A lot, to 4, Not at all) and negative interaction items (e.g., "How often do they criticize you") were measured on a different 4-point scale (from 1, Often, to 4, Never). Positive interaction items were reverse scored before all items were averaged separately for each group (family vs. friends), assuming participants responded to at least $80 \%$ of items. Higher scores reflect greater perceived social support. Participants reported family support within the range of 1.1-4 $(M=3.07, S D=0.33)$ and friend support within the range of 1.3-4 $(M=3.18, S D=0.28)$. Cronbach's alpha for family $(0.84)$ and friend (0.81) suggests high internal reliability.

Feelings regarding school were measured with 10 items taken from the Children of the 90 s survey (University of Bristol, 2020). Participants indicated how true the statements (e.g., "My school is a place where I really like to go each day", "Most of my teachers praise me when I do my schoolwork well", "My school is a place where other schoolmates accept me as I am") were for them on a 3-point scale (from 1, Never/Not True, to 3, Often/Very True). Item responses were averaged if participants responded to at least $80 \%$ of items. Higher average scores indicate more positive feelings toward school. Participants reported school support with the range of $1-3(M=2.33, S D=0.17)$. Cronbach's alpha for school (0.80) suggests high internal reliability of the items. The average scores for each social support measure were used to create a latent trait of adolescent perceptions of social support.

\section{Loneliness, Distress, and Worry}

Three single items measuring student feelings and mood as a response to the COVID-19 pandemic were also informed by COVID surveys previously administered by Generation Scotland (2020). These items were "How many times have you felt lonely or like you didn't have anyone to turn to during the past two weeks?" (1 - None or almost none of the time, to 4, All or almost all of the time), "In the last two weeks, how many times have you felt upset or stressed because of COVID-19?" (1 - Never, to 5, Always), and "Over the last two weeks, how worried have you been about not doing well in school?" (1 - A great deal, to 5, Not at all). These items were used as criterion in our model testing the effects of the social support latent trait, COVID-19 attitudes latent trait, and instruction type on loneliness, distress, and worry. A few more items were included for descriptive purposes: (1) "Over the last two weeks, how worried have you been about getting sick/not seeing friends" with response options "A Great Deal”, "A Lot”, "Some”, "A Little”, and "None At All" and (2) "Think back to before COVID-19, how lonely did you feel" with response options "A lot less lonely before COVID”, "A little less lonely before COVID”, "About the same before COVID", "A little lonelier before COVID", and "A lot lonelier before COVID".

\section{Procedures}

Participants were given the electronic survey link by their teacher as a task to complete, but it was not considered an assignment/homework to allow participants the option to opt-out of the survey. Adolescents completing the survey were first asked questions about demographic information, followed by questions related to COVID-19, and finally questions regarding their perceived social support. Three attention check items were placed roughly after each quartile. To allow flexibility for teachers when giving the survey, participants may have been given the link to complete either at home or during the class period. Therefore, although the survey was not intended to last more than $20 \mathrm{~min}$, participants could have started the survey and completed it over an extended time frame.

\section{Statistical Analyses}

To address the aim of evaluating the current state of adolescent mental and emotional health, frequency and central tendency statistics were obtained using SPSS v26 (IBM). A General Linear Model in SPSS was used to examine the relationship between social support and age before using a 
robust maximum likelihood (MLR) estimation in Mplus v8.5 (Muthén \& Muthén) to address the second aim of evaluating the effect of instruction type on adolescent loneliness, stress, and worry as moderated by protective factors or attitudes towards COVID-19. Because the full model tested the moderating effect of latent traits on observed variables, model fit was determined by comparing to a model without interaction items (Maslowsky et al., 2015).

\section{Results}

\section{Descriptive Statistics}

One-fifth of adolescents (19.3\%) reported that either they or someone they lived with had COVID-19. However, only $61.4 \%$ of these 329 adolescents reporting COVID-19 exposure indicated the diagnosis had been given by a doctor, while the others based a diagnosis on experienced symptoms. Among adolescents without household exposure to COVID-19, 15\% expected someone in their household would probably get COVID-19. Although $31.4 \%$ of adolescents either had household exposure to COVID-19 or expected they were likely to be exposed at some point, $45.4 \%$ acknowledged that it was possible but unlikely someone in their household would get COVID-19 and 23.2\% indicated it was not at all likely someone in their household would get COVID-19.

Participants were also asked to reflect on the ongoing safety measures (e.g., wear a mask, physically distance) to reduce one's risk of exposure to COVID-19. Most adolescents indicated COVID-19 has changed how they spend their day (39.5\% a lot, $26.1 \%$ some), while $22.8 \%$ indicated their lives had only changed a little and $11.6 \%$ noted none at all. Over half (58.5\%) also recognize that COVID-19 is a great deal/a lot harmful to everyone, while another $22.6 \%$ think COVID-19 is somewhat harmful, and only $18.9 \%$ believe COVID-19 is only a little/not at all harmful. Most adolescents $(67.1 \%$ ) recognized that they must (a great deal/a lot) follow safety measures to keep everyone healthy, with only $15.9 \%$ indicating it was somewhat important and $17 \%$ indicating a little or not at all important. Importantly, those adolescents who viewed COVID as more harmful indicated a higher endorsement to following safety measures, $r(1695)=0.47, p<0.001$.

Although some adolescents were concerned a great deal/a lot about the possibility of getting sick (22\%), they were even more concerned about not seeing friends (37.6\%) or not doing well in school (43.8\%). Adolescents were also very lonely based on their responses to the two loneliness items, with over half indicating they felt at least somewhat lonely over the past two weeks $(32.6 \%$ - some of the time; $12.2 \%$ - most of the time; $10.4 \%$-almost all the time) and that compared to prior to COVID-19, they were lonelier now (41.6\% - a lot less lonely before COVID-19; 18.1\% - a little less lonely before COVID-19). While only $18.5 \%$ indicated they were upset or stressed most of the time/always about not seeing people outside their home, this may be because the majority do still regularly leave their home $(60.4 \%$ - at least once per day) and see others when they do (29.7\%big chance of seeing others). However, those who have felt lonely more often $(r=0.20, p<0.001)$ or are lonelier postCOVID-19 $(r=0.15, p<0.001)$ were more worried about not seeing their friends.

\section{Differences in Adolescent Development}

Although there was a difference in perceived family $[F(2,1656)=14.12, M S E=0.33, p<0.001]$ and friend $[F(2,1656)=3.12, M S E=0.29, p=0.044]$ support and, as hypothesized, those in early adolescence indicated higher perceived family support $(M=3.13, S E=0.02)$ compared to middle $(M=3.01, S E=0.02)$ and late $(M=2.95, S E=0.04)$ adolescence, contrary to our hypothesis, higher friend support was also perceived by those in early adolescence $(M=3.20, S E=0.02)$ compared to those in late adolescence $(M=3.11, S E=0.04)$. Because feelings toward school consisted of items regarding the school, teachers, and peers, we had no a priori hypothesis regarding a difference resulting from age. However, consistent with family and friend support, positive feelings towards school $[F(2,1656)=48.57$, $M S E=0.16, p<0.001]$ were higher among those in early adolescence $(M=2.43, S E=0.01)$ compared to those in middle $(M=2.25, S E=0.02)$ and late $(M=2.20, S E=0.03)$ adolescence. Because the pattern of relationship between age and social support was consistent across the social support types, the model was tested in the full sample rather than by each age group.

\section{Effect of Instruction Type of Reaction to COVID-19}

Initially, we expected that both social support and social privileges would have shared variance that represented protective factors. However, model fit $(R M S E A=0.10$, $C F I=0.73, T L I=0.60, S R M R=0.06)$ and low standardized weights indicated items corresponding to social privileges did not share variance with social support. As a result, social privilege items were removed from the model and the latent trait of protective factors, henceforth renamed social support, was then based on only social support. This change resulted in excellent model fit (Fig. 1a). Adolescents' behaviors (e.g., the frequency with which they left their house) and adolescents' perceptions of COVID-19 dangers (e.g., how difficult it is to stay healthy due to COVID-19), were used to construct a latent trait measure of COVID-19 attitudes, which yielded excellent model fit (Fig. 1b). 

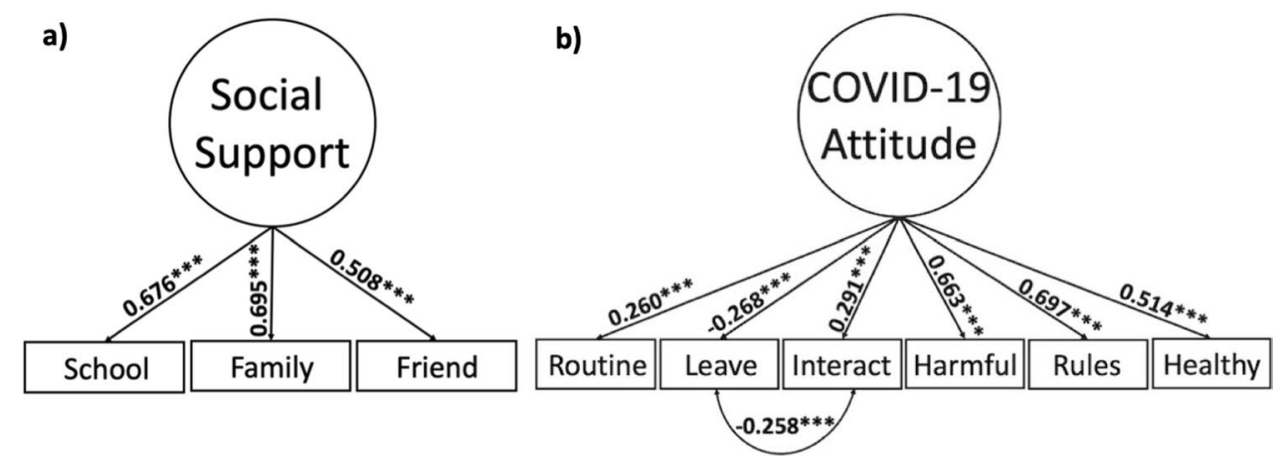

Fig. 1 A Social support latent trait. RMSEA $<0.001 \quad(95 \%$ $\mathrm{CI}=0.000-0.000), \quad \mathrm{CFI}=1.000, \quad \mathrm{TLI}=1.000, \quad \mathrm{SRMR}<0.001$, $N=1703$. "School": Average score of positive feelings toward school items; "Family": Average score of family items on the Perceived Social Support/Conflict Scale; "Friend": Average score of friend items on the Perceived Social Support/Conflict Scale. B COVID attitudes latent trait. $\mathrm{RMSEA}=0.045 \quad(95 \% \mathrm{CI}=0.031-0.061)$, $\mathrm{CFI}=0.973, \quad \mathrm{TLI}=0.949, \quad \mathrm{SRMR}=0.025, \quad N=1699 . \quad$ "Routine": Response to the item "How much has COVID-19 changed how you usually spend your day?" (1 - A lot, to 4, Not at all); "Leave": Response to the item "How many times do you leave your home?"
(1 - Many times per day, to 5, Less than once per week); "Interact": Response to the item "When leaving your home, do you do something with someone who does not live with you?" (1 - I don't leave my home, to 6, I seem someone every time I go out); "Harmful": Response to the item "COVID-19 is harmful to everyone." (1 - A great deal, to 5, Not at all); "Rules": Response to the item "I have to follow rules (wear a mask, stay $6 \mathrm{ft}$ apart, etc.) to keep people from getting COVID-19." (1 - A great deal, to 5, Not at all); "Healthy": "It has become harder to stay healthy because of COVID-19." (1 - A great deal, to 5, Not at all). All estimates are standardized for both latent traits. $p<.05^{*}, p<.01^{* *}, p<.001^{* * *}$

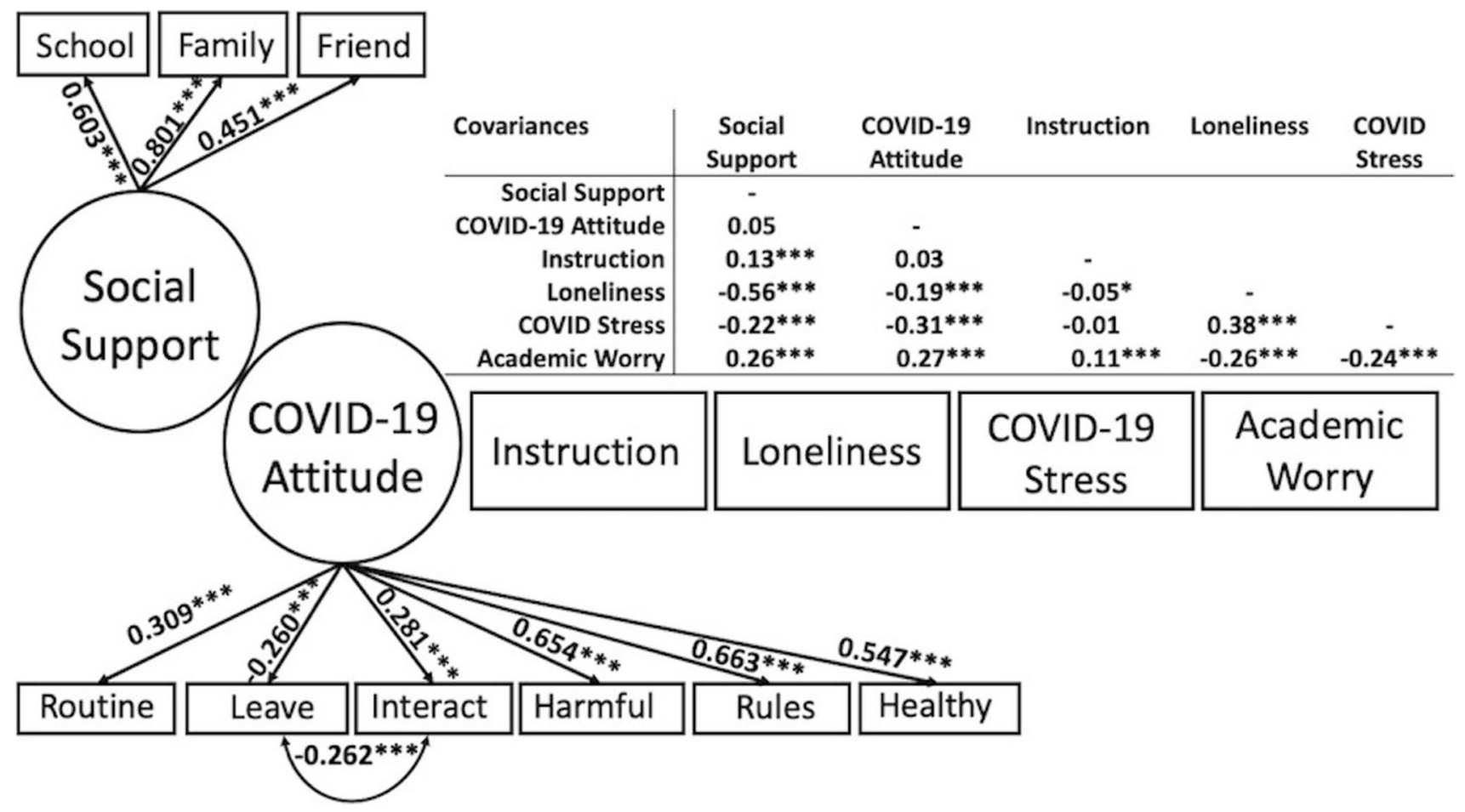

Fig. 2 Measurement model. $\quad X^{2}(53)=321.63, \quad$ RMSEA $=0.054$ $(95 \% \mathrm{CI}=0.049-0.060), \mathrm{CFI}=0.907, \mathrm{TLI}=0.863, \mathrm{SRMR}=0.042$, $N=1685$. "Instruction": Represents whether students indicated if they'd recently been attending school in an all virtual, hybrid, or all in-person format; "Loneliness": "How many times have you felt lonely or like you didn't have anyone to turn to during the past two weeks?" (1 - None or almost none of the time, to 4, All or almost all of the time); "COVID-19 Stress": "In the last two weeks, how many times have you felt upset of stressed because of COVID-19?" (1 Never, to 5, Always); "Academic Worry": "Over the last two weeks, how worried have you been about not doing well in school?" $(1-A$ great deal, to 5, Not at all). All estimates are standardized. $p<.05^{*}$, $p<.01 * *, p<.001 * * *$ 
The measurement model (Fig. 2) and Model 0 (Fig. 3) both demonstrated moderate model fit. The initial model (Model 0) showed that, as hypothesized, both social support and COVID-19 attitudes were significant predictors of adolescent loneliness, COVID-19 stress, and academic worries, such that greater social support and lower COVID-19 concern was associated with lower loneliness, lower stress related to COVID-19, and lower worries related to academics. However, contrary to the hypothesis, instruction type was only related with academic worries, but as expected, those having a greater level of virtual instruction had greater academic worry compared to those receiving greater in-person instruction.
To test the moderating effect of social support and COVID-19 attitude on instruction type, the interaction terms were added to the model. Using chi-square distribution, the log-likelihood ratio test was nonsignificant ( $p>0.05$ ), indicating that Model 1 (Fig. 4) had equivalent fit to Model 0. This model indicated that, although instruction type was not associated with loneliness on its own, that instruction type was moderated by social support, such that the relationship between social support and loneliness became less significant as instruction type moved to more in-person. Meaning, that social support was more important to reducing loneliness when adolescents were receiving a greater level of virtual instruction.
Fig. 3 Model 0. H0 Value $-27,124.11$, Free parameters $=47, X^{2}(55)=341.59$, RMSEA $=0.055(95 \%$ $\mathrm{CI}=0.050-0.061)$, $\mathrm{CFI}=0.900, \mathrm{TLI}=0.859$, SRMR $=0.046, N=1685$. Nonsignificant pathways are estimates are standardized. $p<.05^{*}, p<.01^{* *}, p<.001^{* * *}$ not included in the figure. All

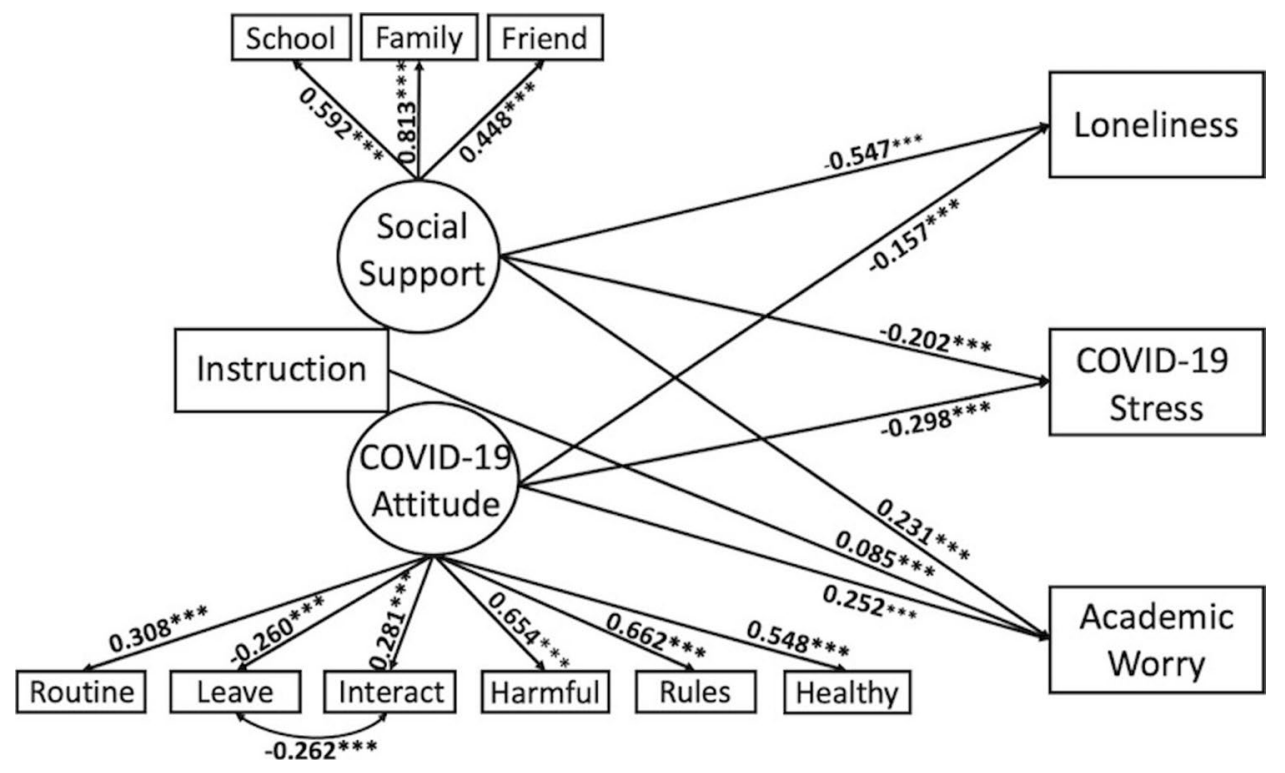

Fig. 4 Model 1. H0 Value $-27,118.88$, Free parameters $=53, N=1685$. Nonsignificant pathways are not included in the figure. All estimates are standardized. $p<.05^{*}$, $p<.01^{* *}, p<.001 * * *$

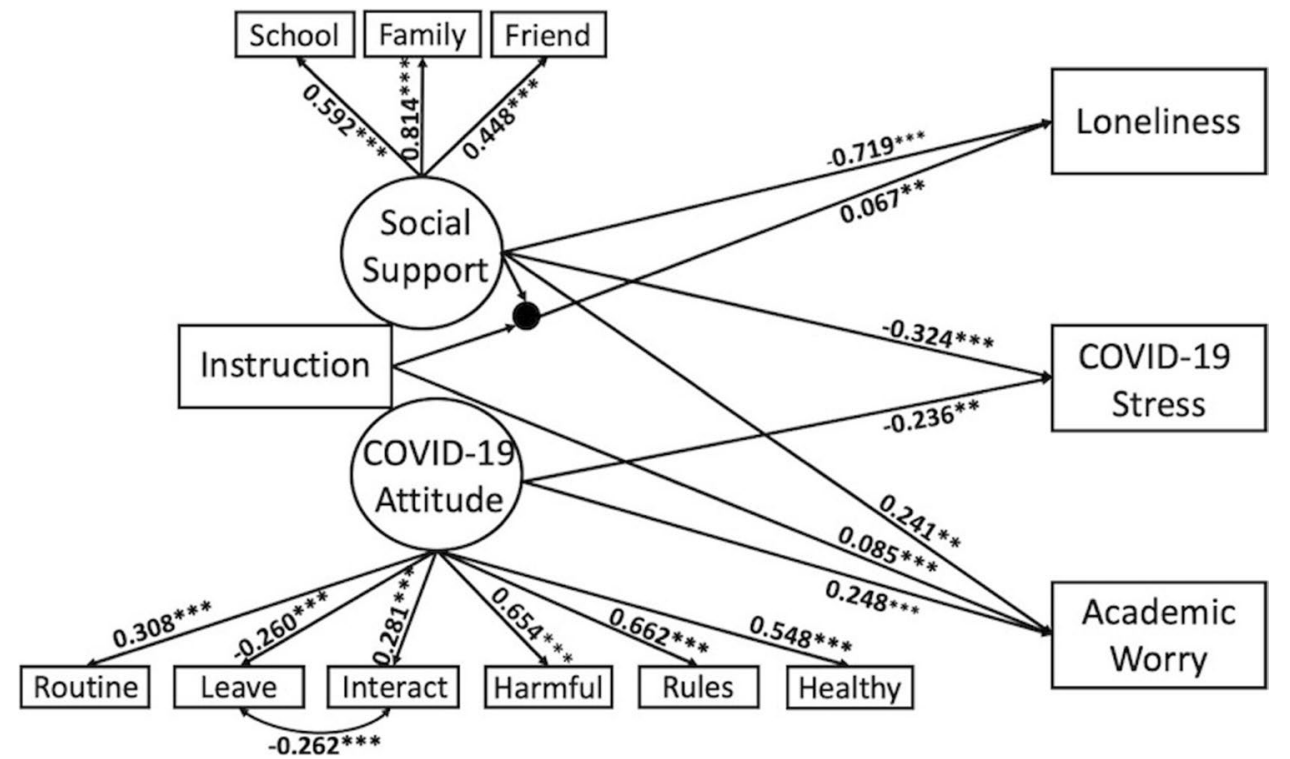




\section{Discussion}

While some previous research has suggested that fear and worry about COVID-19 is associated with increased loneliness (Ellis et al., 2020; Kotwal et al., 2021), our findings suggest that higher endorsement of cautious COVID-19 behaviors and attitudes, which may suggest greater fear, is consistent with other research (Grossman et al., 2021). However, our results indicate that more cautious behaviors and attitudes are associated with greater COVID-19 distress and academic worries. Consistent with research on social support, we also found that higher social support is associated with less loneliness and less COVID-19 distress (Bu et al., 2020; Groarke et al., 2020; Li et al., 2021; Magson et al., 2021), as well as less academic worries ( $\mathrm{Li}$ et al., 2021; Magson et al., 2021; Zhu et al., 2021).

Our findings also suggest that adolescents were still experiencing high levels of loneliness roughly nine months into the pandemic with over 50\% indicating that they were lonelier at that moment than they were prior to the pandemic. The increased loneliness of our sample is consistent with some adult samples (Lee et al., 2020; Ruggieri et al., 2021). However, previous research has mostly focused on the time period when stay-at-home orders were in place. Our findings may suggest that effects of the pandemic on mental health, similar to other epidemics (Loades et al., 2020), could be long-lasting. Although most adolescents may understand the importance of physical distancing, with over half reporting they believe COVID-19 is very harmful and two-thirds reporting they must follow rules (e.g., physical distancing) to keep others safe, they are more concerned about not seeing their friends or doing well in school, which is also consistent with other reports (Scott et al., 2021). These findings suggest that while adolescents understand the importance of safety precautions, the effect of COVID-19 on their emotional wellbeing may be more detrimental.

Initially, stay-at-home orders resulted in high rates of virtual learning, but with the fall 2020 semester, many schools returned to some version of in-person instruction, either fully in-person or a hybrid method. There was preference for those in childhood and earlier adolescence to return to in-person instruction due to lower incidence of COVID-19 in younger individuals (Center for Disease Control, 2021b), with a greater emphasis of hybrid methods for the older adolescents. Indeed, in our sample, $69 \%$ of those in early adolescence had returned to all in-person instruction, compared to only $20 \%$ in late adolescence. Given the high reported frequency of loneliness and concern for seeing friends, there may be concern that taking proactive steps toward safety against COVID-19 is increasing risk of mental health issues. However, when examining the effect of instruction type on loneliness, COVID-19 stress, and academic worries, increased virtual instruction was only associated with academic worries directly, and loneliness when combined with lower perceived social support. This moderating effect is the first to be reported, demonstrating the importance of comparing different types of instruction on mental health as we progress through the pandemic and beyond, should schools continue to have online options.

Overall, our findings may suggest that although loneliness is common during the pandemic, being back to in-person instruction may not be enough to alleviate feelings of loneliness. Even though individuals are in the same space as their peers, they may still be faced with physical distancing guidelines and an underlying pandemic-related stress. With vaccines becoming available for everyone, we may expect that loneliness and other mental health issues could decline. However, we are likely a long way from returning to a similar state as that prior to the pandemic given that COVID19 vaccines are not utilized by a majority in all states and new variants are showing increased transmission. Therefore, efforts to address loneliness and stress are needed. Such efforts may include adjusting one's mindset regarding situational control (Milman et al., 2020), mindfulness training (Yuan, 2021), increased access to counseling through one's school (Anderson et al., 2020), or increasing perceptions of social support (Saltzman et al., 2020).

\section{Study Limitations}

While these findings are the first to demonstrate in a U.S. sample of adolescents that the importance of social support for COVID-19 related stress and loneliness varies based on the amount of online education, they should be considered in light of limitations. Although instruction type had a small effect on academic concerns and loneliness when moderated by social support, it is possible the effect could have been larger if our sample included a larger population of those receiving all virtual instruction. Despite our large overall sample size $(N=1,708)$ and a likely accurate representation of the current distribution of instruction type, most of the sample was receiving hybrid instruction $(42 \%)$ or all in-person instruction (54\%), and therefore, findings may not be representative of those receiving all virtual instruction. Additionally, while longitudinal studies (Lee et al., 2020; Ruggieri et al., 2021) suggest that loneliness increased from pre- to post-COVID-19 pandemic, our sample is from only one time point. However, because the effects of the pandemic are expected to be long-lasting (Loades et al., 2020), it is important to have reports of wellbeing at various time points throughout the pandemic to provide converging evidence. 
Our choices of measurement could also be a potential limitation. Because younger adolescents often cannot accurately report their family's SES by indicators of income, employment, or education level, and because all adolescents in our sample were from schools identified as Title I schools (i.e., at least $40 \%$ of the student body are from low-income families; U.S., Department of Education, 2021) we utilized a subjective measure of social status (Goodman et al., 2011) to allow students to report how they believe their social status compares to others. Relatedly, investigations of the effects of COVID-19 have been rapid since the start of the pandemic with substantial knowledge adding to our understanding with each passing month. As a result, measures of COVID-19 risk (Jaspal et al., 2020; Yildirim \& Guler, 2020), preventative behavior (Breakwell et al., 2021), and fear (Ahorsu et al., 2020) have been validated in adults and/or after the design and implementation of our current study. Therefore, single items from previous mass surveys of COVID-19 to measure COVID-19 attitudes and behaviors were used instead for our study. Although, these were only single items, they were similar to items on validated measures, were also similar to other studies involving adolescent samples (Dumas et al., 2020; Graupensperger et al., 2020; Murata et al., 2020; Rogers et al., 2021; Taylor et al., 2020), and allowed us to keep questions simple and the study brief, which was important given the age range of our sample.

\section{Conclusions and Broader Implications}

In conclusion, although virtual instruction is associated with academic worries, returning to in-person instruction does not alleviate loneliness and distress brought on by the pandemic. Instead, a focus on increasing the social support provided to adolescents, as well as promoting confidence and self-efficacy may be more beneficial. Only time will tell how long-lasting the effects of the pandemic are, but through a greater understanding of adolescent perceptions we can hope to improve adolescent wellbeing more effectively. More broadly, adults may also continue to struggle with loneliness and distress although they are returning to the workforce. The pandemic and the collective trauma experienced by people of all ages is likely to persist. Future research should continue to examine the long-term effect and intergenerational impact that the stress from the pandemic has on psychological wellbeing (Bowers \& Yehuda, 2016). As our study and others that have examined natural disasters and world events that resulted in collective trauma, it is possible the pandemic will have lasting negative effects on mental health for children, adolescents, adults, and future generations, beyond the official ending (Esterwood \& Saeed, 2020).
Author Contributions All authors contributed to the study conception, design, and data collection. Data analyses and preparing the original draft of the manuscript was performed by CCC and JMG reviewed and revised the initial version. All authors read and approved the final manuscript.

Data Availability The dataset generated and analyzed for the current study is available from the corresponding author upon reasonable request.

\section{Declarations}

Ethics The study was approved by the IRB and was performed in accordance with ethical standards. No identifying details of participants were collected during the study and participants gave informed assent for voluntary participation and publication, and legal guardians were given the opportunity to opt-out their child, in accordance with our study's IRB approval.

Informed Consent Participants gave informed assent for voluntary participation and publication, and legal guardians were given the opportunity to opt-out their child, in accordance with our study's IRB approval.

Conflicts of Interest The authors have no relevant financial or nonfinancial interests to disclose.

\section{References}

Ahorsu, D. K., Lin, C. Y., Imani, V., Saffari, M., Griffiths, M. D., \& Pakpour, A. H. (2020). The fear of COVID-19 scale: Development and initial validation. International Journal of Mental Health and Addition. Epub ahead of print 27 Mar. https://doi.org/10.1007/ s11469-020-00270-8

American Psychological Association (2021, February 25). What did distance learning accomplish? http://www.apa.org/monitor/2020/ 09/distance-learning-accomplish

Anderson, H., Haeder, S., Caseman, K., Mathur, A., \& Ulmen, K. (2020). When adolescents are in school during COVID-19, coordination between school-based health centers and education is key. Journal of Adolescent Health, 67, 745-746. https://doi.org/ 10.1016/j.jadohealth.2020.09.005

AP News. (2021, March 15). Is it safe to reopen schools during the pandemic? https://apnews.com/article/virus-outbreak-ap-topnews-health-ap-fact-check-public-health-9acd2a3f7af12421a4f3 9572afe5baac

Barenbaum, E., \& Smith, T. (2016). Social support as a protective factor for children impacted by HIV/AIDS across varying living environments in southern Africa. AIDS Care, 28, 92-99. https:// doi.org/10.1080/09540121.2016.1176683

Breakwell, G. M., Fino, E., \& Jaspal, R. (2021). Preventive behaviors index: Development and validation in two samples from the United Kingdom. Evaluation and the Health Professions, 44, 77-86. https://doi.org/10.1177/0163278720983416

Breakwell, G. M., \& Jaspal, R. (2020). Identity change, uncertainty and mistrust in relation to fear and risk of COVID-19. Journal of Risk Research. Epub ahead of print.

Bowers, M., \& Yehuda, R. (2016). Intergenerational transmission of stress in humans. Neuropharmacology, 41, 232-244. https://doi. org/10.1038/npp.2015.247

Bu, F., Steptoe, A., \& Fancourt, D. (2020). Loneliness during a strict lockdown: Trajectories and predictors during the COVID-19 pandemic in 38,217 United Kingdom adults. Social Science \& 
Medicine, 265, 113521. https://doi.org/10.1016/j.socscimed.2020. 113521

Cavanaugh, C. (2001). The effectiveness of interactive distance education technologies in K-12 learning: A meta-analysis. International Journal of Educational Telecommunication, 7, 73-78.

Cavanaugh, A. M., \& Buehler, C. (2015). Adolescent loneliness and social anxiety: The role of multiple sources of support. Journal of Social and Personal Relations, 33, 149-170. https://doi.org/10. $1177 / 0265407514567837$

Center for Disease Control. (2021a, February 18). Risk for COVID-19 infection, hospitalization, and death by age group. https://www. cdc.gov/coronavirus/2019-ncov/covid-data/investigations-disco very/hospitalization-death-by-age.html

Center for Disease Control. (2021b, March 19). Operational strategy for K-12 schools through phased mitigation. https://www.cdc.gov/ coronavirus/2019-ncov/community/schools-childcare/operationstrategy.html\#learning-modes

Chowkwanyun, M., \& Reed, A., Jr. (2020). Racial health disparities and Covid-19 - caution and context. New England Journal of Medicine, 383, 201-203. https://doi.org/10.1056/NEJMp2012910

Clouston, S., Natale, G., \& Link, B. G. (2021). Socioeconomic inequalities in the spread of coronavirus-19 in the United States: A examination of the emergence of social inequalities. Social Science \& Medicine, 268, 113554. https://doi.org/10.1016/j.socsc imed.2020.113554

Cohen, S., \& Wills, T. A. (1985). Stress, social support, and the buffering hypothesis. Psychological Bulletin, 98(2), 310-357. https:// doi.org/10.1037/0033-2909.98.2.310

Duan, L., Shao, X., Wang, Y., Huang, Y., Miao, J., Yang, X., \& Zhu, G. (2020). An investigation of mental health status of children and adolescents in china during the outbreak of COVID-19. Journal of Affective Disorders, 275, 112-118. https://doi.org/10.1016/j. jad.2020.06.029

Dumas, T. M., Ellis, W., \& Litt, D. M. (2020). What does adolescent substance use look like during the COVID-19 pandemic? Examining changes in frequency, social contexts, and pandemic-related predictors. Journal of Adolescent Health, 67, 354-361. https:// doi.org/10.1016/j.jadohealth.2020.06.018

Ellis, W. E., Dumas, T. M., \& Forbes, L. M. (2020). Physically isolated but socially connected: Psychological adjustment and stress among adolescents during the initial COVID-19 crisis. Canadian Journal of Behavioural Science, 52, 177-187. https://doi.org/10. 1037/cbs0000215

Esterwood, E., \& Saeed, S. A. (2020). Past epidemics, natural disasters, Covid-19, and mental health: Learning from history as we deal with the present and prepare for the future. Psychiatric Quarterly, 91, 1121-1133. https://doi.org/10.1007/s11126-020-09808-4

Gausman, J., \& Langer, A. (2020). Sex and gender disparities in the COVID-19 Pandemic. Journal of Women's Health, 29, 465-466. https://doi.org/10.1089/jwh.2020.8472

Generation Scotland, The University of Edinburgh. (2020, April) CovidLife. https://www.ed.ac.uk/generation-scotland/for-researchers/ covidlife

Goodman, E., Adler, N. E., Kawachi, I., Frazier, A. L., Huang, B., \& Colditz, G. A. (2011). Adolescents' perceptions of social status: Development and evaluation of a new indicator. Pediatrics, 108(2), e31. https://doi.org/10.1542/peds.108.2.e31

Graupensperger, S., Benson, A. J., Kilmer, J. R., \& Evans, M. B. (2020). Social (un)distancing: Teammate interactions, athletic identity, and mental health of student-athletes during the COVID19 pandemic. Journal of Adolescent Health, 67, 662-670. https:// doi.org/10.1016/j.jadohealth.2020.08.001

Groarke, J. M., Berry, E., Graham-Wisener, L., McKenna-Plumley, P. E., McGlinchey, E., \& Armour, C. (2020). Loneliness in the UK during the COVID-19 pandemic: Cross-sectional results from the COVID-19 psychological wellbeing study. PLoS One, 15(9), e0239698. https://doi.org/10.1371/journal.pone.0239698

Grossman, E. S., Hoffman, Y. S. G., Palgi, Y., \& Shrira, A. (2021). COVID-19 related loneliness and sleep problems in older adult: Worries and resilience as potential moderators. Personality and Individual Differences, 168, 110371. https://doi.org/10.1016/j. paid.2020.110371

Harper, C. A., Satchell, L. P., Fido, D., \& Latzman, R. D. (2020). Functional fear predicts health compliance in the COVID-19 pandemic. International Journal of Mental Health and Addiction. https://doi. org/10.1007/s11469-020-00281-5

Jaspal, R., \& Breakwell, G. M. (2020). Socio-economic inequalities in social network, loneliness and mental health during the COVID19 pandemic. International Journal of Social Psychiatry. Epub ahead of print. https://doi.org/10.1177/0020764020976694

Jaspal, R., Fino, E., \& Breakwell, G. M. (2020). The COVID-19 own risk appraisal scale (CORAS): Development and validation in two samples from the United Kingdom. Journal of Health Psychology. Epub ahead of print 28 Oct. https://doi.org/10.1177/13591 05320967429

Kingery, J. N., Erdley, C. A., \& Marshall, K. C. (2011). Peer acceptance and friendship as predictors of early adolescents' adjustment across the middle school transition. Merrill-Palmer Quarterly, 57, 215-243. https://doi.org/10.1353/mpq.2011.0012

Kotwal, A. A., Holt-Lunstad, J., Newmark, R. L., Cenzer, I., Smith, A. K., Covinsky, K. E., Escueta, D. P., Lee, J. M., \& Perissinotto, C. M. (2021). Social isolation and loneliness among San Francisco bay area older adults during the COVID-19 shelter-in-place orders. Journal of the American Geriatrics Society, 69, 20-29. https://doi.org/10.1111/jgs.16865

Lahav, Y. (2020). Psychological distress related to COVID-19: The contribution of continuous traumatic stress. Journal of Affective Disorders, 277, 129-137. https://doi.org/10.1016/j.jad.2020.07. 141

Lee, C. M., Cadigan, J. M., \& Rhew, I. C. (2020). Increases in loneliness among young adults during the COVID-19 pandemic and association with increases in mental health problems. Journal of Adolescent Health, 67, 714-717. https://doi.org/10.1016/jadoh ealth.2020.08.009

Li, X., Tang, X., Wu, H., Sun, P., Wang, M., \& Li, L. (2021). Covid-19 related stressors and Chinese adolescents' adjustment: The moderating role of coping and online learning satisfaction. Frontiers in Psychiatry, 12, 633523. https://doi.org/10.3389/fpsyt.2021. 633523

Loades, M. E., Chatburn, E., Higson-Sweeney, N., Reynolds, S., Shafran, R., Brigden, A., Linney, C., McManus, M. N., Borwick, C., \& Crawley, E. (2020). Rapid systematic review: The impact of social isolation and loneliness on the mental health of children and adolescents in the context of COVID-19. Journal of the American Academy of Child and Adolescent Psychiatry, 59, 1218-1239. https://doi.org/10.1016/j.jaac.2020.05.009

Magson, N. R., Freeman, J. A., Rapee, R. M., Richardson, C. E., Oar, E. L., \& Fardouly, J. (2021). Risk and protective factors for prospective changes in adolescent mental health during the Covid-19 pandemic. Journal of Youth and Adolescence, 50, 44-57. https:// doi.org/10.1007/s10964-020-01332-9

Malhi, G. S., Das, P., Bell, E., Mattingly, G., \& Mannie, Z. (2019). Modelling resilience in adolescence and adversity: A novel framework to inform research and practice. Translational Psychiatry, 9, 316. https://doi.org/10.1038/s41398-019-0651-y

Maslowsky, J., Jager, J., \& Hemken, D. (2015). Estimating and interpreting latent variable interactions: A tutorial for applying the latent moderated structural equations method. International Journal of Behavioral Development, 39, 87-96. https://doi.org/ $10.1177 / 0165025414552301$ 
Milman, E., Lee, S. A., \& Neimeyer, R. A. (2020). Social isolation as a means of reducing dysfunctional coronavirus anxiety and increasing psychoneuroimmunity. Brain, Behavior, and Immunity, 87, 138-139. https://doi.org/10.1016/j.bbi.2020.05.007

Munasinghe, S., Sperandei, S., Freebairn, L., Conroy, E., Jani, H., Marjanovic, S., \& Page, A. (2020). The impact of physical distancing policies during the COVID-19 pandemic on health and well-being among Australian adolescents. Journal of Adolescent Health, 67, 653-661. https://doi.org/10.1016/j.jadohealth.2020.08.008

Murata, S., Rezeppa, T., Thoma, B., Marengo, L., Krancevich, K., Chiyka, E., Hayes, B., Goodfriend, E., Deal, M., Zhong, Y., Brummit, B., Coury, T., Riston, S., Brent, D. A., \& Melhem, N. M. (2020). The psychiatric sequelae of the COVID-19 pandemic in adolescents, adults, and health care workers. Depression and Anxiety, 38, 233-246. https://doi.org/10.1002/da.23120

National Institute on Aging. Midlife in the United States II (MIDUS II) Self- administered Questionnaire 2, Items L11a. - L111., J2a. $-\mathrm{J} 2 \mathrm{j} ., \mathrm{J} 4 \mathrm{a}$. - J4h., which correspond to items 1a.-11., 2a.-2j., and 3a. - 3h., respectively, in the protocol, 2002.

Nelson, E. E., Jarcho, J. M., \& Guyer, A. E. (2016). Social re-orientation and brain development: An expanded and updated view. Developmental Cognitive Neuroscience, 17, 118-127. https://doi. org/10.1016/j.den.2015.12.008

Rogers, A. A., Ha, T., \& Ockey, S. (2021). Adolescents' perceived socio-emotional impact of COVID-19 and implications for mental health: Results from a U.S.-based mixed-methods study. Journal of Adolescent Health, 68, 43-52. https://doi.org/10.1016/j.jadoh ealth.2020.09.039

Ruggieri, S., Ingoglia, S., Bonfanti, R. C., \& Lo Coco, G. (2021). The role of online social comparison as a protective factor for psychological wellbeing: A longitudinal study during the COVID-19 quarantine. Personality and Individual Differences, 171, 110486. https://doi.org/10.1016/j.paid.2020.110486

Saltzman, H., Hansel, T. C., \& Bordnick, P. S. (2020). Loneliness, isolation, and social support factors in post-COVID-19 mental health. Psychological Trauma, 12, S55-S57. https://doi.org/10. 1037/tra0000703

Scott, S. R., Rivera, K. M., Rushing, E., Manczak, E. M., Rozek, C. S., \& Doom, J. R. (2021). "I hate this": A qualitative analysis of adolescents' self-reported challenges during the COVID-19 pandemic. Journal of Adolescent Health, 68, 262-269. https:// doi.org/10.1016/j.jadohealth.2020.11.010

Shachar, M., \& Neumann, Y. (2003). Differences between traditional and distance education academic performances: A meta-analytic approach. The International Review of Research in Open and Distributed Learning, 4, 73-88. https://doi.org/10.19173/irrodl. v4i2.153

Schacter, H. L., \& Margolin, G. (2019). The interplay of friends and family in adolescents' daily lives: Towards a dynamic view of social support. Social Development, 28, 708-724. https://doi.org/ $10.1111 /$ sode. 12363

Shah, S. M. A., Mohammad, D., Qureshi, M. F. H., Abbas, M. Z., \& Aleem, S. (2021). Prevalence, psychological responses and associated correlates of depression, anxiety and stress in a global population, during the coronavirus disease (COVID-19) pandemic. Community Mental Health Journal, 57, 101-110. https://doi.org/ 10.1007/s10597-020-00728-y
Steinberg, L. (2005). Cognitive and affective development in adolescence. Trends in Cognitive Sciences, 9, 69-74. https://doi.org/10. 1016/j.tics.2004.12.005

Taylor, S., Landry, C. A., Paluszek, M. M., Feregus, T. A., McKay, D., \& Asmundson, G. J. G. (2020). COVID stress syndrome: Concept, structure, and correlates. Depression and Anxiety, 37, 706-714. https://doi.org/10.1002/da.23071

Uink, B. N., Modecki, K. L., \& Barber, B. L. (2017). Disadvantaged youth report less negative emotion to minor stressors when with peers: An experience sampling study. International Journal of Behavioral Development, 41, 41-51. https://doi.org/10.1177/ 0165025415626516

University of Bristol. (2020). COVID-19: Making a difference. http:// www.bristol.ac.uk/alspac/covid-19/

U.S. Department of Education (2021, November 22). Improving Basic Programs Operated by Local Educational Agencies (Title I. Part A). https://www2.ed.gov/programs/titleiparta/index.html

Vijayakumar, N., \& Pfeifer, J. H. (2020). Self-disclosure during adolescence: Exploring the means, targets, and types of personal exchanges. Current Opinion in Psychology, 31, 135-140. https:// doi.org/10.1016/j.copsyc.2019.08.005

Virtanen, T. E., Vasalampi, K., Kiuru, N., Lerkkanen, M. K., \& Poikkeus, A. M. (2020). The role of perceived social support as a contributor to the successful transition from primary to lower secondary school. Scandinavian Journal of Educational Research, 64, 967-983. https://doi.org/10.1080/00313831.2019.1639816

Weine, S. M., Ware, N., Hakizimana, L., Tugenberg, T., Currie, M., Dahnweih, G., Wagner, M., Polutnik, C., \& Wulu, J. (2014). Fostering resilience: Protective agents, resources, and mechanisms for adolescent refugees' psychosocial well-being. Adolesc Psychiatry (hilversum), 4, 164-176. https://doi.org/10.2174/2210676604 03140912162410

World Health Organization (2021, February 25). Weekly epidemiological update. https://www.who.int/publications/m/item/covid-19weekly-epidemiological-update

Wu, T., Jia, X., Shi, H., Niu, J., Yin, X., Xie, J., \& Wang, X. (2021). Prevalence of mental health problems during the COVID-19 pandemic: A systematic review and meta-analysis. Journal of Affective Disorders, 281, 91-98. https://doi.org/10.1016/j.jad.2020.11. 117

Yildirim, M., \& Guler, A. (2020). Factor analysis of the COVID-19 perceived risk scale: A preliminary study. Death Studies. Epub ahead of print 25 Jun. https://doi.org/10.1080/07481187.2020. 1784311

Yuan, Y. (2021). Mindfulness training on the resilience of adolescents under the COVID-19 epidemic: A latent growth curve analysis. Personality and Individual Differences, 172, 110560. https://doi. org/10.1016/j.paid.2020.110560

Zhu, S., Zhuang, Y., \& Ip, P. (2021). Impacts on children and adolescents' lifestyle, social support, and their association with negative impacts of the Covid-19 pandemic. International Journal of Environmental Research and Public Health, 18, 4208. https://doi. org/10.3390/ijerph18094780

Publisher's note Springer Nature remains neutral with regard to jurisdictional claims in published maps and institutional affiliations. 